\section{Deux théorèmes sur les familles de transformations.}

$$
\text { Par }
$$

\section{Wa cław Sierpiński (Warszawa).}

Le but de cette Note est de démontrer à l'aide de l'axiome du choix les deux théorèmes suivants:

Théorème $I . S i M$ est un ensemble infini de puissance met $F^{H}$ une famille de puissance $m$ de fonctions définies dans $M$ (dont les valeurs peuvent appartenir à $M$ ou non), ì existe une famille $\Phi$ formée de $2^{\mathrm{m}}$ sous-ensembles distincts de $\mathbb{M}$ telle qu'on a

$$
f(E) \neq \dot{H} \text { pour } \quad E \in \Phi, \quad H \in \Phi, E \neq H, \quad f \in F .
$$

Théorème II. Si $M$ est un ensemble infini de puissance $m$ et $F$ une famille de puissance $m$ de fonctions définies dans $M I$ et prenant (pour les éléments de $M$ ) chaque valeur de $M$ au plus une fois (pouvant d'ailleurs prendre, pour les éléments de $M$, des valeurs étrangères à $M$ ), il existe une famille $\Phi$ formée de $2^{\mathrm{m}}$ sous-ensembles distincts de M telle qu'on a

$$
\left.f(E)-H \neq 0 \text { pour } E \in \Phi, H \in \Phi, \quad E \neq H, f \in F^{1}\right) .
$$

Le théorème I a été énoncé sans démonstration par $\Lambda$. Lindenbaum en $1937^{2}$ ); sa démonstration ne fut pas publiée et elle m'est inconnue. Après que j'ai trouvé la démonstration du théorème I, le théorème II fut démontré par une autre voie par M. Kuratowski $\left.{ }^{3}\right)$.

1) C., c. Kuratowski, Topologie I (Monografie Matematyezne t. III, Warszawa-Lwów 1933), p. 218.

2) Ann. Soc. Polonaise Math. 15, p. 185.

3) Voir la Note qui va suivre de M. Kuratowski.
Démonstration du théorème I. Soit $M$ un ensemble infini de puissance $m, F$ une famille de puissance $m$ de fonctions définies dans $M$ (à valeurs quelconques). Soit $\varphi$ le plus petit nombre ordinal de puissance m; l'axiome du choix implique l'existence d'une suite transfinie du type $q$,

$$
x_{1}, x_{2}, \ldots, x_{\omega}, x_{\omega+1}, \ldots, x_{\xi}, \ldots
$$

formée de tous les éléments distincts de l'ensemble $M$.

Soit $F_{1}$ la famille de toutes les fonctions $f(x)$ de la famille $F$ jouissant de la propriété $P$ suivante:

$P$. Il existe un sous-ensemble $M_{f}$ de $M$ de puissance $\mathrm{m}$, tel que $f(x) \neq x$ et $f(x) \in M$ pour $x \in M_{f}$ et que $f(x) \neq f\left(x^{\prime}\right)$ pour $x \in M_{f}$, $x^{\prime} \in M_{P}, x \neq x^{\prime}$.

La famille $F_{1}$ est évidemment de puissance $\leqslant \mathfrak{m}$; il existe done une suite transtinie du type $\%$,

$$
f_{1}(x), f_{2}(x), \ldots, f_{\omega}(x), f_{\omega+1}(x), \ldots, f_{\xi}(x), \ldots
$$

formée de fonctions de la famille $F_{1}$ et dans laquelle toute fonction de la famille $F_{1}$ figure au moins une fois.

Nous définirons maintenant par l'induction transfinie deux suites transfinies du type $\varphi$

$$
\begin{gathered}
p_{1}, p_{2}, \ldots, p_{\omega}, p_{\omega+1}, \ldots, p_{\xi}, \ldots \\
q_{1}, q_{2}, \ldots, q_{\omega}, q_{\omega+1}, \ldots, q_{\xi}, \ldots
\end{gathered}
$$

comme il suit. Soit $p_{1}=x_{1}$ et $q_{1}=x_{2}$. Soit maintenant $\alpha$ un nombre ordinal $<\varphi$ et supposons que nous avons déjà défini tous les éléments $p_{\xi}$ et $q_{\xi}$, où $\xi<2 \alpha$ (ce qui a lieu pour $\alpha=1$ ). Soit $P_{2 \alpha}$, resp. $Q_{2 \alpha}$ l'ensemble de tous éléments $p_{\xi}$, resp. $q_{\xi}$, où $\xi<2 \alpha$; vu que $\alpha<\varphi$, on a $\overline{\bar{P}}_{2 \alpha}<m$ et $\overline{\bar{Q}}_{2 c}<\mathrm{m}$. La fonction $f_{a}$ étant à valeurs distinctes dans l'ensemble $M_{f_{\alpha}}$ qui est de puissance $m$ et les ensembles $P_{2 \alpha}$ et $Q_{2 \alpha}$ étant de puissances $<\mathrm{m}$, il existe des termes $x_{\xi}$ de la suite (3) tels que $x_{\xi} \epsilon M_{f_{c}}-\left(P_{2 \alpha}+Q_{2 \alpha}\right)$ et $f_{a}\left(x_{\xi}\right)$ non $\epsilon P_{2 \alpha} ;$ nous définirons $p_{2 \alpha}$ comme le premier parmi eux, nous poserons $q_{2 \alpha}=f_{a}\left(p_{2 \alpha}\right)$, nous définirons ensuite $p_{\text {. }}$ comme le premier terme $x_{\xi}$ de la suite (1) tel que $x_{\xi} \in M_{f_{c \alpha}}-\left(P_{2 a}+Q_{2 \alpha}\right), x_{\xi} \neq p_{2 \alpha}, x_{\xi} \neq q_{2 \alpha}, f_{a c}\left(x_{\xi}\right)$ non $\epsilon P_{2 \alpha}$ et tel que $x_{\xi} \in M_{f_{c}}-\left(P_{2 a}+Q_{2 \alpha}\right), x_{\xi}+p_{2 \alpha}, x_{\xi}+q_{2 \alpha}$, et nous poserons $q_{2 \alpha+1}=f_{\alpha}\left(p_{2 \alpha+1}\right)$. Les suites transfinies (5) et (6) sont ainsi définies par l'induction transfinie et on voit nies (5) peine que les termes de la suite (5) sont tous distincts et distincts de termes de la suite (6). 
Comme $\mathrm{m}^{2}=\mathrm{m}$ (ce qui résulte de l'axiome du choix), l'ensemble $T$ de tous les nombres ordinaux $<\varphi$ peut être décomposé en $m$ ensembles disjoints de puissance $m, T_{\xi}(\xi<\varphi)$. Soit $U$ un sousensemble quelconque de $T$ et désignons par $E_{U}$ l'ensemble de tous les éléments $p_{2 c+1}$, où $a \in \sum_{\xi \in U} T_{\S}$, et de tous les éléments $p_{2 c}$, où a non $\epsilon \sum_{\Sigma \in U} T_{\Xi}$. Je dis que la famille $\Phi=\left\{E_{U}\right\}_{U \subset T}$ satisfait au théorème $I$.

Soit, en effet, $U \subset T, V \subset T, U \neq T$. Un des ensembles $U$ et $V$ contient done un nombre ordinal $\eta$ qui n'appartient pas à l'autre. Si $\eta \in U-\mathrm{F}$, on a $p_{2 \alpha+1} \in E_{U}-E_{V}$ pour $\alpha \in T_{\eta}$ (vu que les ensembles $T_{\xi}(\xi<\varphi)$ sont disjoints et que les termes de la suite (5) sont distincts) et, si $\eta \in T-U$, on a $p_{z_{\alpha}} \in E_{U}-E_{\mathrm{V}}$ pour $a \in T_{\eta}$. vu que $\overline{\overline{\bar{T}_{\eta}}}=\mathrm{mt}$, on a done toujours

$$
\overline{\overline{E_{U}-\overline{E_{L}}}}=\mathrm{m} \text { pour } \quad U \subset T, V \subset T, U \neq T .
$$

Anx sous-ensembles distincts de $T$ correspondent donc des ensembles distincts $E_{U}$; vu que $\overline{\bar{T}}=\mathrm{m}$, nous concluons que la famille $\phi$, est de puissance $2^{\mathrm{m}}$ et on a

$$
\overline{E-\bar{H}}=\mathfrak{m} \quad \text { pour } \quad E \in \Phi, H \in \Phi, E \neq H .
$$

Soit maintenant $f$ une fonction donnée de la famille $F$ et distinguons deux cas: $f \in F-F_{1}$ et $f \in F_{1}$.

Soit $f \in F-F_{1}$. Désignons par $R$ l'ensemble de tous les éléments $x$ de $M$ tels que $f(x) \neq x$ et soit $s$ l'ensemble de tous les éléments $f(x)$, où $x \in R$, c. à d. posons $S=f(R)$. Si l'ensemble $S M$ est de puissance $\mathrm{m}$, il existe, comme on voit sans peine, un ensemble $M_{f} \subset R$ de puissance $m$, tel que $f(x) \neq f\left(x^{\prime}\right)$ pour $x \in M_{f}, x^{\prime} \in M_{f}, \dot{x} \neq x^{\prime}$; la fonction $f$ jouit donc de la propriété $P$, ce qui est imposible, puisque $f \in F-F_{1}$. L'ensemble $S M$ est done de puissance $<\mathrm{m}$. Soit $E$ un sous-ensemble de $M$ : Si $y \in M f(E)-E$, on a $y \in M$ et $y=f(x)$, où $x \in E$, et $\{y$ non $\epsilon E$, donc $f(x) \neq x$, d'où $x \in R$ et $y=f(x) \in f(R)=S$, done $y \in M S$. On a ainsi $M f(E)-E \subset M S$, done $\overline{\boldsymbol{M} f(E)-\bar{E}}<m$ pour $E C M$. Soit maintenant $E$ un ensemble de la famille $\Phi$ et supposons que $f(E)=H \in \Phi$; vu que $H \subset M$ (pour $H \epsilon \Phi)$, on aurait done $M f(E)-E=H-E$, done $\overline{\overline{H-E}}<\mathfrak{m}$, ce qui est impossible pour $E \neq H$, d'après ( 7 ). On a donc pour $f \epsilon F-F_{1}^{\prime}$ l'inégalité (1).
Soit maintenant $f \in F_{1}$; il existe done un nombre ordinal $a<\varphi$ tel que $f=f_{\alpha}$. Soient $U$ et $F$ deux sous-ensembles (distincts ou non) de $T$. Si $\alpha \in \sum_{\xi \in U} T_{\xi}$, on a (vu la définition de l'ensemble $E_{U}$ ) $p_{2 a+1} \in E_{U}$ et $q_{2 a+1}=f_{c}\left(p_{2 a+1}\right) \in f_{c}\left(E_{U}\right)$, done, ru que $E_{V}$ est formé d'éléments de la suite (5) qui sont tous $\neq q_{2 \alpha+1}$, on a $f_{a}\left(E_{U}\right)-E_{V} \neq 0$. Si $\alpha$ non $\epsilon \sum_{\xi \in U} T_{\xi}$, on a $p_{2 \alpha} \in E_{U}$ et $q_{2 \alpha}=f_{\alpha}\left(p_{2 \alpha}\right) \in f_{\alpha}\left(E_{U}\right)$, d'où encore $f_{a}\left(E_{U}\right)-E_{V} \neq 0$.

On a done toujours

$$
f_{a}(E)-H \neq 0 \text { pour } E \epsilon \Phi, H \in \Phi, a<\varphi .
$$

Comme $f=f_{a}$, on a donc, à plus forte raison, linégalité (1).

L'inégalité (1) subsiste done toujours et le théorème I est ainsi démontré.

Démonstration du théorème II. Reprenons la démonstration du théorème I, mais supposons que les fonctions de la famille $F$ premnent chaque valeur de $M$ au plus une fois. Distinguons deux cas: $f \in F-F_{1}$, et $f \in \mathscr{F}_{1}$.

Soit $f \in F-F_{1}$ et supposons qu'il existe des ensembles $E \in \Phi$ et $H \in \Phi$ tels que $E \neq H$ et $f(\boldsymbol{E}) \subset H$. Comme $f(\boldsymbol{E} R) \subset f(\boldsymbol{E}) \subset H \subset M$ et $f \in F-F_{1}$, on a $\overline{\overline{f(E \bar{R})}}<\mathrm{m}$ et, la fonction $t$ prenant chaque valeur de $M$ au plus une fois, on en conclut que $\overline{\overline{E R}}<\mathrm{m}$. Or, il résulte de la définition de l'ensemble $R$ que $E-R=f(E-R)$, d'où

done

$$
\begin{gathered}
E-R \subset(E-R)+f(R E)=f(E-R)+f(R E)=f(E) \subset H, \\
E-H \subset E R \text { et } \overline{\overline{E-H}} \leqslant \overline{\overline{E R}}<\mathrm{m},
\end{gathered}
$$

contrairement à $(7)$. On a done dans notre cas l'inégalité $\left.(2)^{1}\right)$. Soit maintenant $f \in F_{1}$; il existe donc un nombre ordinal $a<\varphi$ tel que $f=f_{c}$; d'après (8) on a donc l'inégalité 2 ).

l'inégalité (2) a donc lieu toujours et le théorème II est démontré. Il a des applications importantes dans la théorie des ensembles de points.

1) Cette partie de la démonstration du théorème II est due à M. R. Sikorski.

Fondamenta Mathematicae. T. XXXIV. 